\title{
Real and complex variable positive definite functions
}

\section{Jorge Buescu}

Dep. Matemática

FCUL and CMAF

Portugal

E-mail address: jbuescu@ptmat.fc.ul.pt

\section{A. C. Paixão}

Área Departamental de Matemática

ISEL

Portugal

E-mail address: apaixao@dem.isel.ipl.pt

\begin{abstract}
In this paper we present an overview of the implications of our previously derived results for positive definite kernels on the general theory of positive definite functions. We begin by exploring the consequences of a set of differential inequalities on the global behaviour of a smooth positive definite function of one real variable. Then we propose a natural extension of this study to the complex variable case and derive consequences of positive definiteness for meromorphic functions.
\end{abstract}

\section{Positive definite functions}

The purpose of this paper is to present a survey of some consequences of previously derived results on the theory of positive definite kernels for the field of positive definite functions. This a review paper; detailed proofs may be found where indicated.

Received by the editors 2013-04-08.

2010 Mathematics Subject Classification. Primary: 42A82. Secondary: 30A10, 30C40.

Key words: positive definite functions, analytic functions, complex analysis, inequalities.

The first author acknowledges partial support by Fundação para a Ciência e Tecnologia, PEst-OE/MAT/UI0209/2011. 
We deal mostly with issues of global regularity and analiticity of positive definite functions and with the development of a coherent theory of positive definiteness of functions of a complex variable. To this end, a brief review of the properties of real variable positive definite functions relevant for our purposes will be performed below.

A function $f: \mathbb{R} \rightarrow \mathbb{C}$ is positive definite if

$$
\sum_{j, k=1}^{n} f\left(x_{j}-x_{k}\right) \xi_{j} \overline{\xi_{k}} \geq 0
$$

for every choice of $x_{1}, \ldots x_{n} \in \mathbb{R}$ and $\xi_{1}, \ldots, \xi_{n} \in \mathbb{C}$; that is, if every square matrix $\left[f\left(x_{j}-x_{k}\right)\right]_{j, k=1}^{n}$ is positive semidefinite.

Let us state what we shall call the basic properties of positive definite functions:

(1) $f(0) \geq 0$;

(2) $f(-x)=\overline{f(x)}$ for all $x \in \mathbb{R}$;

(3) $|f(x)|^{2} \leq f(0)^{2}$.

These arise simply from consideration of the cases $n=1,2$ in (1.1). It will be useful for future reference to stress that no hypothesis on $f$ besides positive definiteness is necessary for these basic properties to hold. In particular, no regularity assumptions, not even continuity of $f$, are required.

Positive definite functions have for long been known to satisfy the following characterization.

Theorem 1.1 (Bochner). A continuous function $\phi: \mathbb{R} \rightarrow \mathbb{C}$ is positive definite if and only if it is the Fourier transform of a finite positive measure $\mu$ on $\mathbb{R}$, that is

$$
\phi(x)=\int_{-\infty}^{+\infty} e^{-i t x} d \mu(t)
$$

From the Bochner integral representation (1.2) the definition of positive definite function (1.1) is easily derived. We stress that, for real variable functions, (1.2) and (1.1) are equivalent. The Bochner representation is the most expedite way to prove that a function is positive definite, as is the case of the following examples:

(1) $f_{1}(x)=\cos (a x)$;

(2) $f_{2}(x)=e^{-|x|}$;

(3) $f_{3}(x)=\frac{1}{1+x^{2}}$;

(4) $f_{4}(x)=\max (0,1-|x|)$;

(5) $f_{5}(x)=e^{-x^{2}}$. 


\section{Positive definite kernels}

In order to establish a new family of properties of positive definite functions, it will be essential to consider them from another point of view.

Given a set $E$, a positive definite matrix in the sense of Moore (see e.g. Moore [21], Aronszajn [1]) is a function $k: E \times E \rightarrow \mathbb{C}$ such that

$$
\sum_{j, k=1}^{n} k\left(x_{j}, x_{k}\right) \xi_{j} \overline{\xi_{k}} \geq 0
$$

for all $n \in \mathbb{N},\left(x_{1}, \cdots, x_{n}\right) \in E^{n}$ and $\left(\xi_{1}, \cdots, \xi_{n}\right) \in \mathbb{C}^{n}$; that is, all finite square matrices $M$ of elements $m_{i j}=k\left(x_{i}, x_{j}\right), i, j=1, \ldots, n$, are positive semidefinite.

From (2.1), it is easily shown that a positive definite matrix in the sense of Moore has the following properties:

(1) $k(x, y)=\overline{k(y, x)}$ for all $x, y \in E$;

(2) $k(x, x) \geq 0$ for all $x \in E$;

(3) $|k(x, y)|^{2} \leq k(x, x) k(y, y)$ for all $x, y \in E$.

Since these $k$ frequently appear as kernels of positive definite integral operators on $I \subseteq \mathbb{R}$, they are more commonly known as positive definite kernels, and we shall henceforth refer to them as such. We shall refer to properties (1) - (3) above as the basic properties of positive definite kernels, noting again that also here these properties hold without any regularity hypotheses on $k$.

We remark that the theorem of Moore-Aronszajn ([1], [21]) provides an equivalent characterization of positive definite kernels as reproducing kernels, of which the most widely studied is the classical Bergman kernel. Since we shall not need this approach we omit a more detailed discussion.

The following definition is useful in the study of differential properties of positive definite kernels $k$ defined in $I \subset \mathbb{R}$.

Definition 2.1. Let $I \subset \mathbb{R}$ be an open interval. A function $k: I^{2} \rightarrow \mathbb{C}$ is said to be of class $\mathcal{S}_{n}\left(I^{2}\right)$ if, for every $m_{1}=0,1, \ldots n$ and $m_{2}=0,1, \ldots n$, the partial derivatives $\frac{\partial^{m_{1}+m_{2}}}{\partial y^{m_{2}} \partial x^{m_{1}}} k(x, y)$ exist and are continuous in $I^{2}$.

Class $\mathcal{S}_{n}\left(I^{2}\right)$ is thus the weakest differentiability class where equality of all mixed partial derivatives up to order $n$ in each variable is ensured.

The next two results have been proved in various degrees of generality $[5,6,7]$. 
Theorem 2.2. Let $I \subseteq \mathbb{R}$ be an interval and $k(x, y)$ be a positive definite kernel of class $\mathcal{S}_{n}\left(I^{2}\right)$. Then, for all $0 \leq m \leq n$,

$$
k_{m}(x, y) \equiv \frac{\partial^{2 m}}{\partial y^{m} \partial x^{m}} k(x, y)
$$

is a positive definite kernel of class $\mathcal{S}_{n-m}\left(I^{2}\right)$.

Theorem 2.3. Let $I \subseteq \mathbb{R}$ be an interval and $k(x, y)$ be a positive definite kernel of class $\mathcal{S}_{n}\left(I^{2}\right)$. Then, for all integers $m_{1}, m_{2}$ with $0 \leq m_{i} \leq n, i=$ 1,2 , and all $x, y \in I$ we have

$$
\left|\frac{\partial^{m_{1}+m_{2}}}{\partial y^{m_{2}} \partial x^{m_{1}}} k(x, y)\right|^{2} \leq \frac{\partial^{2 m_{1}}}{\partial y^{m_{1}} \partial x^{m_{1}}} k(x, x) \frac{\partial^{2 m_{2}}}{\partial y^{m_{2}} \partial x^{m_{2}}} k(y, y) .
$$

In $[5,6,7]$ these results have been proved directly, using appropriate finite difference matrices and the positive semidefiniteness condition (2.1). We should observe however that both results admit proofs in the more abstract setting of the associated reproducing kernel Hilbert space; indeed, Theorem 2.3 may be interpreted as a generalized Cauchy-Schwartz inequality in this setting. These results have useful applications in the theory of integral equations: for instance, they supply an appropriate integrability condition and ensure compactness for positive operators on unbounded domains, as well as allowing for refinements in the eigenvalue distribution of such operators $[6,8]$.

Positive definite kernels and positive definite functions are closely related. Indeed, if $f: \mathbb{R} \rightarrow \mathbb{C}$ is a positive definite function, then $k(x, y)=f(x-y)$ is a positive definite kernel in $\mathbb{R}$, as is clear from the corresponding definitions.

This very simple observation allows us to derive immediately the basic properties (1) - (3) of positive definite functions described in $\S 1$ from the corresponding basic properties (1) - (3) for positive definite kernels. This relation has, however, deeper consequences: results valid for positive definite kernels translate into corresponding properties of positive definite functions. To be specific, the counterparts of Theorems 2.2 and 2.3 for positive definite functions have been established in [9] under the appropriate assumptions and are stated below.

Proposition 2.4. Let $f: \mathbb{R} \rightarrow \mathbb{C}$ be a positive definite function and suppose $f$ is of class $C^{2 n}$ in some neighborhood of the origin for some positive integer $n$. Then $f \in C^{2 n}(\mathbb{R})$ and for all integers $m$ with $0 \leq m \leq n$, the function $f_{m} \equiv(-1)^{m} f^{(2 m)}$ is positive definite.

This property, derived in [9] by the above correspondence, is well-known, even in somewhat more general settings (see e.g. [22], pg 140). It may 
be derived for instance from Bochner's representation (1.2) by formally differentiating an even number of times under the integral sign [13].

The counterpart of Theorem 2.3 gives rise to a two-parameter family of differential inequalities for positive definite functions.

Proposition 2.5. Let $f: \mathbb{R} \rightarrow \mathbb{C}$ be a positive definite function and suppose $f$ is of class $C^{2 n}$ in some neighborhood of the origin for some positive integer $n$. Then $f \in C^{2 n}(\mathbb{R})$ and for all integers $m_{1}, m_{2}$ with $0 \leq m_{i} \leq n, i=1,2$ and every $x \in \mathbb{R}$ we have

$$
\left|(-1)^{m_{2}} f^{\left(m_{1}+m_{2}\right)}(x)\right|^{2} \leq(-1)^{m_{1}+m_{2}} f^{\left(2 m_{1}\right)}(0) f^{\left(2 m_{2}\right)}(0) .
$$

Remark 2.6. Since both $(-1)^{m_{1}} f^{\left(2 m_{1}\right)}$ and $(-1)^{m_{2}} f^{\left(2 m_{2}\right)}$ are positive definite functions by Proposition 2.4, the right hand side of (2.3) is necessarily non-negative, thus ensuring that the corresponding inequality is meaningful.

\section{Global properties of differentiable PDFs}

It is possible to show that the case $n=3$ in (1.1) implies that, if $f$ is continuous in a neighbourhood of the origin, then it is uniformly continuous in $\mathbb{R}$ (see e.g. [3], Corollary 1.4.10). We shall see that this result is in some sense prototypical of positive definite functions: condition (1.1) implies some sort of local-to-global "propagation of regularity" from (a neighborhood of) the origin to $\mathbb{R}$, of which this is the $C^{0}$ case. These propagation of regularity results are well-known, although exact priorities have been difficult to assign.

Theorem 3.1 (propagation of regularity). Let $f$ be a positive definite function. Suppose $f$ is of class $C^{2 k}$ in a neighbourhood of the origin. Then $f$ is $C^{2 k}(\mathbb{R})$.

The most direct route to prove this result is probably to use the Bochner representation (1.2) and the methods of Harmonic Analysis; see e.g. Donoghue [14]. We thus see that the propagation of continuity mentioned above is just the $k=0$ case of this theorem.

Two remarks are in order. In the first place, notice that the propagation of differentiability only occurs for even-order derivatives. In fact, it is shown in [9] that even-order derivatives play a determining role for positive definite functions, in contrast to odd-order derivatives. In the second place, the analogous result holds for $C^{\infty}$ functions; indeed, it holds for the analytic case, as can be seen in e.g. [4, 16]. These results have been recently extended to quasianalytic classes of positive definite functions and distributions and more elaborate classes of differentiability $[11,12,20]$. The basic phenomenon is however the same: regularity in a neighbourhood of 
the origin propagates as the corresponding regularity to the whole of $\mathbb{R}$. The positivity condition (1.1) may thus be seen as effectively coupling the local behavior at the origin with the global behavior.

One of the basic properties of positive definite functions described in $\S 1$ is that $|f(x)| \leq f(0)$ for every $x \in \mathbb{R}$, which implies that $f$ vanishes identically if $f(0)=0$. The next result, which is a consequence of Proposition 2.5, may be viewed as the extension of this property for differentiable positive definite functions.

Theorem 3.2. Let $f: \mathbb{R} \rightarrow \mathbb{C}$ be a positive definite function and suppose $f$ is of class $C^{2 n}$ in a neighborhood of the origin for some non-negative integer $n$. If $f^{(2 m)}(0)=0$ for some non-negative integer $m \leq n$, then $f$ is constant on $\mathbb{R}$.

Proof. See [9].

Remark 3.3. Trivially, a constant function is positive definite if and only if it is non-negative, so the constant in the above theorem must be nonnegative.

Remark 3.4. Notice that for the special case $n=0$, the statement of the theorem follows from basic property (3) in $\S 1$.

Remark 3.5. An immediate consequence of Theorem 3.2 is that the only positive definite polynomials are the non-negative constants.

Remark 3.6. The positive definite function $f(x)=\left(1+x^{2}\right)^{-1}$ supplies a simple example of the fact that no analog of Theorem 3.2 exists for oddorder derivatives.

Implications of the differential inequalites (2.2) on the global analiticity of a positive definite function will be stated in the next Theorem. For this purpose, it will be useful to recall the following characterization of real-analytic functions.

Lemma 3.7. Let $f$ be a real function in $\mathbb{C}^{\infty}(I)$ for some open interval $I$. Then $f$ is real analytic if and only if, for each $\alpha \in I$, there are an open interval $J$, with $\alpha \in J \subset I$, and constants $C>0$ and $R>0$ such that the derivatives satisfy

$$
\left|f^{(k)}(x)\right| \leq C \frac{k !}{R^{k}} \quad \forall x \in J .
$$

Proof. This is a standard result; see e.g. [18], Proposition 1.2.12.

Remark 3.8. Although for convenience the result is stated for real functions, it extends in the obvious way to the present context of complex-valued functions of a real variable. 
Theorem 3.9. Let $f: \mathbb{R} \rightarrow \mathbb{C}$ be a positive definite function and suppose $f$ is of class $C^{\infty}$ in a neighborhood of the origin. Then, if there exist positive constants $M$ and $D$ such that

$$
0 \leq(-1)^{n} f^{(2 n)}(0) \leq D \frac{(2 n) !}{M^{2 n}}
$$

for every non-negative integer $n$, we have:

i) $f$ is analytic in $\mathbb{R}$;

ii) Let $l=\limsup \sqrt[2 n]{\frac{\left|f^{(2 n)}(0)\right|}{(2 n) !}}$. Then $l<\infty$. Defining $h=1 / l$ if $l \neq$ 0 and $h=\infty$ if $l=0$, there exist $\alpha, \beta \in[h,+\infty]$ such that $f$ extends holomorphically to the complex strip $\{z \in \mathbb{C}:-\alpha<z<\Im(z)<\beta\}$, where $\alpha$ and $\beta$ are maximal with this property. Moreover, if $h<\infty$, $f$ cannot be holomorphically extended to both the points $z=i$ and $z=-i$ simultaneously, implying in particular that $h=\min \{\alpha, \beta\}$.

Proof. See [9].

Remark 3.10. Observe that if equality in the left hand side of (3.1) holds for some $n$, then $f$ is constant by Theorem 3.2. In this case every statement above holds trivially.

Remark 3.11. Theorem 3.9 derives its conclusions from direct application of the inequalities provided by Proposition 2.5 to positive definite functions satisfying a suitable set of hypotheses on the existence and growth of even order derivatives at the origin. There exist in the literature results closely related to these, which have been obtained under different assumptions and through the use of distinct methods. We mention, in particular, those stated in [3] and [19] where the assertions ii) in Theorem 3.9 under the (stronger) hypothesis that $f$ is analytic in a neighbourhood of the origin.

Incidentally, these results may be used together with the conclusions of part i) of Theorem 3.9 to refine the statements of part ii) of the Theorem, namely by adding the fact that the holomorphic extension of $f$ to the maximal strip must present singularities at both points $z=-i \alpha$ and $z=i \beta$ whenever $\alpha$ or $\beta$ are finite.

\section{From real to complex variables}

As stated at the outset, one of the purposes of this paper is to present a coherent theory of complex-variable positive definite functions. The appropriate definition of positive definiteness is not a priori obvious in the complex setting (see e.g. [7] for a basic discussion). Some light into this problem is however shedded by the following considerations. 
A real-analytic positive definite function $f: \mathbb{R} \rightarrow \mathbb{C}$ (for instance, a function in the conditions of Theorem 3.9) extends holomorphically to a horizontal strip of the complex plane $\mathcal{A}=\{z \in \mathbb{C}:-\alpha<\Im(z)<\beta\}$, with $\alpha, \beta>0$, as described above. It is also possible to show [3] that in this case the Bochner integral representation (1.2) extends holomorphically to the whole strip $\mathcal{A}$ by the integral representation

$$
f(z)=\int_{-\infty}^{+\infty} e^{-i t z} d \mu(t)
$$

Using this representation one may easily conclude that $f(z)$ satisfies

$$
\sum_{i, j=1}^{n} f\left(z_{i}-\overline{z_{j}}\right) \xi_{i} \overline{\xi_{j}} \geq 0
$$

for all $n \in \mathbb{N}$ and for any collections $\left\{z_{i}\right\}_{i=1, \ldots, n} \subset \mathbb{C}$ such that $z_{i}-\overline{z_{j}} \in \mathcal{A}$ for all $i, j=1, \ldots, n$ and $\left\{\xi_{i}\right\}_{i=1, \ldots, n} \in \mathbb{C}$.

Let $\Omega=\left\{z \in \mathbb{C}:-\frac{\alpha}{2}<\Im(z)<\frac{\beta}{2}\right\}$. Defining $k(z, u)=f(z-\bar{u})$, it is immediate to observe that $k$ is a sesquianalytic function (i.e., separately analytic in $z$ and anti-analytic in $u$ ) in $\Omega^{2}$. Then, with the above definition, condition (4.1) assumes the form

$$
\sum_{i, j=1}^{n} k\left(z_{i}, z_{j}\right) \xi_{i} \overline{\xi_{j}} \geq 0
$$

for all $n \in \mathbb{N},\left\{\xi_{i}\right\}_{i=1, \ldots, n} \in \mathbb{C}$ and $\left\{z_{i}\right\}_{i=1, \ldots, n} \subset \Omega$. Thus $k$ is a holomorphic positive definite kernel in $\Omega$. These kernels are known in the functional analytic setting, for historical reasons, as holomorphic reproducing kernels. We shall use both terminologies interchangeably, depending on the context.

Holomorphic reproducing kernels satisfy the following basic properties, corresponding to their real counterparts in $\S 2$ (see e.g. [7]):

(1) $k(z, u)=\overline{k(u, z)}$ for all $z, u \in \Omega$;

(2) $k(z, z) \geq 0$ for all $z \in \Omega$;

(3) $|k(z, u)|^{2} \leq k(z, z) k(u, u)$ for all $z, u \in \Omega$.

Notice that, as in the real case, these properties follow directly from (4.2), with no requirements on regularity of $k$. This fact suggests that, if we define positive definiteness in the complex plane starting from (4.1), holomorphy of $f$, and thus sesquiholomorphy of $k$, should not be a priori requirements.

In fact, if sesquiholomorphy of $k$ holds, much stronger statements are valid. The following differential properties of holomorphic reproducing kernels are proved in [7] in the context of several complex variables, $\mathbb{C}^{2 n}$. 
Since only the case $n=1$ will be used in this paper, the corresponding results (stated there as Theorem 3.4, Corollary 3.5 and Theorem 3.6) will be adapted to this case.

Theorem 4.1. Let $\Omega \subset \mathbb{C}$ be an open set and $k: \Omega^{2} \rightarrow \mathbb{C}$ be a holomorphic reproducing kernel on $\Omega$. Then, for any positive integer $m \in \mathbb{N}$,

$$
k_{m}(z, u) \equiv \frac{\partial^{2 m}}{\partial \bar{u}^{m} \partial z^{m}} k(z, u)
$$

is a holomorphic reproducing kernel on $\Omega$.

Proof. See [7].

Corollary 4.2. Let $\Omega \subset \mathbb{C}$ be an open set and $k: \Omega^{2} \rightarrow \mathbb{C}$ be a holomorphic reproducing kernel on $\Omega$. Then for all $z, u \in \Omega$ and all $m \in \mathbb{N}$ we have

$$
\begin{aligned}
\frac{\partial^{2 m}}{\partial \bar{u}^{m} \partial z^{m}} k(z, z) & \geq 0 \text { and } \\
\left|\frac{\partial^{2 m}}{\partial \bar{u}^{m} \partial z^{m}} k(z, u)\right|^{2} & \leq \frac{\partial^{2 m}}{\partial \bar{u}^{m} \partial z^{m}} k(z, z) \frac{\partial^{2 m}}{\partial \bar{u}^{m} \partial z^{m}} k(u, u) .
\end{aligned}
$$

Theorem 4.3. Let $\Omega \subset \mathbb{C}$ be an open set and $k: \Omega^{2} \rightarrow \mathbb{C}$ be a holomorphic reproducing kernel on $\Omega$. Then for all $m_{1}, m_{2} \in \mathbb{N}$ and all $z, u \in \Omega$ we have

$$
\left|\frac{\partial^{m_{1}+m_{2}}}{\partial \bar{u}^{m_{2}} \partial z^{m_{1}}} k(z, u)\right|^{2} \leq \frac{\partial^{2 m_{1}}}{\partial \bar{u}^{m_{1}} \partial z^{m_{1}}} k(z, z) \frac{\partial^{2 m_{2}}}{\partial \bar{u}^{m_{2}} \partial z^{m_{2}}} k(u, u) .
$$

Proof. See [7].

Notice that the second inequality in Corollary 4.2 is an automatic consequence (basic property (3)) of positive definiteness of $k_{m}$, and is simultaneously the special case $m_{1}=m_{2}=m$ in (4.3).

Remark 4.4. We observe that the statement of Theorems 4.1, 4.3 and Corollary 4.2 differ from the corresponding results in [7] in the fact that the former are stated for open sets while the latter were stated for domains. A simple analysis of the proofs of Theorems 4.1, 4.3 and Corollary 4.2 shows that these results are purely local and connectedness is irrelevant, so that the results above are valid as stated here.

In the next section we shall define what is meant by a complex variable positive definite function exploring precisely this relationship with holomorphic reproducing kernels. 


\section{Complex variable positive definite functions}

We begin by presenting a definition which will prove extremely useful in what follows.

Definition 5.1. A set $S \subset \mathbb{C}$ is called a codifference set if there exists a set $\Omega \subset \mathbb{C}$ such that $S$ may be written as

$$
S=\Omega-\bar{\Omega} \equiv\left\{z \in \mathbb{C}: \exists z_{1}, z_{2} \in \Omega: z=z_{1}-\overline{z_{2}}\right\} .
$$

If $S$ is a codifference set and $\Omega \subset C$ is as in Definition 5.1, we shall say that $S=\operatorname{codiff}(\Omega)$. Note that the set operation used in (5.1) is not the usual set difference.

If $S=\operatorname{codiff}(\Omega)$ with $\Omega \neq \emptyset$, it follows easily from Definition 5.1 that $S$ has nonempty intersection with the imaginary axis, is symmetric with respect to it and $\Omega$ is not uniquely determined by $S$. Simple examples of codifference sets include the hereby called horizontal strips defined by

$$
S\left(r, \alpha_{1}, \alpha_{2}\right)=\left\{z=a+b i \in \mathbb{C}:|a|<r, \alpha_{1}<b<\alpha_{2}\right\}
$$

with $r, \alpha_{1}, \alpha_{2}$ positive real or infinite. It is readily seen that $S\left(r, \alpha_{1}, \alpha_{2}\right)=$ $\operatorname{codiff}\left(S\left(r / 2, \alpha_{1} / 2, \alpha_{2} / 2\right)\right)$. It is also easily seen that codifference sets need not be simply connected or even connected [10].

Definition 5.2. A function $f: \mathbb{C} \rightarrow \mathbb{C}$ is said to be positive definite in $S \subset$ $\mathbb{C}$ if, for every $n \in \mathbb{N}$ and every finite collection $\left\{z_{k}\right\}_{k=1, \ldots, n}$ such that $z_{i}-$ $\overline{z_{j}} \in S$ for all $i, j=1, \ldots, n$ (or, equivalently, such that codiff $\left\{z_{k}\right\}_{k=1, \ldots, n} \subset$ $S$ ), we have

$$
\sum_{i, j=1}^{n} f\left(z_{i}-\overline{z_{j}}\right) \xi_{i} \overline{\xi_{j}} \geq 0
$$

for every collection of $\xi_{i} \in \mathbb{C}$.

Remark 5.3. We stress once more that Definition 5.2 does not require any regularity on the function $f$, not even continuity. The properties of positive definite functions we prove throughout this section are valid in this setting.

Holomorphic extensions of real-analytic positive definite functions obtained by complex analytic extension of the Bochner representation, as in (4.1), provide examples of complex positive definite functions on a strip containing the real axis in $\mathbb{C}$. A very different phenomenon occurs with the function $f(z)=\frac{1}{\cosh \left(\frac{\pi z}{2}\right)}$. This function is positive definite in any horizontal strip of the form

$$
s_{n}=S(\infty, 4 n-1,4 n+1)=\{z \in \mathbb{C}:(4 n-1) i<z<(4 n+1) i, \quad n \in \mathbb{Z}\} .
$$


In fact, $f$ admits the well-known integral representation (see e.g. [17])

$$
f(z)=\frac{1}{\cosh \left(\frac{\pi z}{2}\right)}=\int_{-\infty}^{\infty} \frac{1}{\cosh t} e^{-i z t} d t
$$

on the strip $s_{0}=S(\infty,-1,1)=\{z \in \mathbb{C}:|\Im z|<1\}$, which implies that it is positive definite on $s_{0}$. However, the function $f$ is periodic of period $4 i$ and therefore positive definite in every strip $s_{n}$, even though the integral representation (5.2) is divergent outside $S_{0}$. This is a phenomenon not unlike holomorphic extension of functions defined by power series outside the circle of convergence. Of course, as previously observed, all the horizontal strips $s_{n}$ are codifference sets.

Remark 5.4. It is interesting to note the following fact. Let $f$ be a positive definite function in $S \subset \mathbb{C}$ and suppose $S$ contains a horizontal line $\{z \in \mathbb{C}$ : $z=x+i b, x \in \mathbb{R}\}$ for some $b \in \mathbb{R}$. Define $F: \mathbb{R} \rightarrow \mathbb{C}$ by $F(x)=f(x+i b)$. It is then easily seen that $\sum_{i, j=1}^{n} F\left(x_{i}-x_{j}\right) \xi_{i} \overline{\xi_{j}} \geq 0$ for any collections $\left\{x_{k}\right\}_{k=1, \ldots, n} \subset \mathbb{R}$ and $\left\{\xi_{k}\right\}_{k=1, \ldots, n} \subset \mathbb{C}, n \in \mathbb{N}$, and therefore $F$ is a positive definite function on $\mathbb{R}$. On the other hand, if $S$ contains the imaginary axis and we define $G(x)=f(i x)$ we get, for the same collections, the related condition $\sum_{i, j=1}^{n} G\left(x_{i}+x_{j}\right) \xi_{i} \overline{\xi_{j}} \geq 0$. In a more abstract, grouptheoretic approach, this condition is referred to as positive-definiteness in the semigroup sense, while the usual condition of positive-definiteness (1.1) corresponds to positive-definiteness in the group sense; see e.g. [2].

Two basic properties of complex variable positive definite functions may be easily derived from Definition 5.2.

Proposition 5.5 (Positivity on the imaginary axis). Suppose that $f$ is positive definite in $S$. Then $f(i b) \geq 0$ for all $i b \in \operatorname{codiff}(S)$, where $b \in \mathbb{R}$.

Proof. See [10].

Thus, consideration of order 1 matrices allows us to conclude that $f$ is real and non-negative in every point of the imaginary axis.

Proposition 5.6 (Basic inequalities on the complex plane). Suppose that $f$ is positive definite in $S$. Let $a, b, \beta \in \mathbb{R}$ and suppose that $S$ contains the points $a+b i,-a+b i,(b+\beta) i$ and $(b-\beta) i$. Then

$$
\begin{gathered}
f(-a+b i)=\overline{f(a+b i)} ; \\
|f(a+b i)|^{2} \leq f(i(b+\beta)) f(i(b-\beta))
\end{gathered}
$$

Proof. See [10].

Complex positive definite functions defined on codifference sets are, to a large extent, controlled by their behaviour on the imaginary axis, as illustrated by the following results. 
Lemma 5.7. Suppose $S$ is a set of complex numbers such that $S \cap \Im(z)=i I$ for some real interval $I$, and let $f: S \rightarrow \mathbb{C}$ be a positive definite function. Then:

(1) if $f(i u)=0$ for some $u \in I$, then $f(i c)=0$ for all $c \in \operatorname{int}(I)$;

(2) if $f(i u) \neq 0$ for every $u \in I$, then $g=\log f$ is mid-point convex on iI, that is

$$
g\left(\frac{i b_{1}+i b_{2}}{2}\right) \leq \frac{g\left(i b_{1}\right)+g\left(i b_{2}\right)}{2}
$$

for every $b_{1}, b_{2} \in I$.

Proof. See [10].

Remark 5.8. Convexity of $g=\log f$ on the imaginary axis seems to have been first proved by Dugué [15] under the assumption that $f$ is holomorphic. As shown in this lemma, midpoint convexity of $g$ on the imaginary axis always holds without any assumptions on regularity of $f$.

Theorem 5.9. Suppose $S$ is an open codifference set and let $f: S \rightarrow \mathbb{C}$ be a positive definite function. If $f$ has a zero on every connected component of $S \cap \Im(z)$, then $f$ vanishes identically on $S$.

Proof. See [10].

\section{Meromorphic positive definite functions}

As already suggested in $\S 4$, positive definite functions are related with positive definite kernels in two complex variables in the following way. Suppose $f$ is positive definite in $S \subset \mathbb{C}$ and that $V=\left\{(z, u) \in \mathbb{C}^{2}: z-\bar{u} \in S\right\}$. Then $k(z, u) \equiv f(z-\bar{u})$ defines a function $k: V \rightarrow \mathbb{C}$. Let $\Omega \in \mathbb{C}$ such that $\Omega^{2} \subset V$ or, equivalently, that $\operatorname{codiff}(\Omega) \subset S$. If $\left\{z_{k}\right\}_{k=1, \ldots, n} \subset \Omega$ for $n \in \mathbb{N}$, it follows that codiff $\left\{z_{k}\right\}_{k=1, \ldots, n} \subset \operatorname{codiff}(\Omega) \subset S$. Therefore

$$
\sum_{i, j=1}^{n} k\left(z_{i}, z_{j}\right) \xi_{i} \overline{\xi_{j}} \geq 0
$$

for all $\xi_{i} \in \mathbb{C}, i=1, \ldots, n$, and $k$ is a positive definite kernel in $\Omega$.

Suppose now that $S$ and $\Omega$ are open sets and that $f$ is holomorphic in $S$. Then $k$ is sesquiholomorphic in $\Omega^{2}$ and is thus a holomorphic positive definite kernel in $\Omega$.

This relation between complex positive definite functions and complex positive definite kernels allows us to establish important results about the former derived from known results about the latter. 
Theorem 6.1. Let $S \subset \mathbb{C}$ be an open set and suppose that $f: S \rightarrow \mathbb{C}$ is positive definite and holomorphic in $S$. Then $f_{m}(z) \equiv(-1)^{m} f^{(2 m)}(z)$ is a positive definite function in $S$ for every $m \in \mathbb{N}$.

Proof. See [10].

Theorem 6.2. Let $S \subset \mathbb{C}$ be an open set and suppose that $f: S \rightarrow \mathbb{C}$ is a holomorphic positive definite function. Suppose that $S$ contains the points $a+b i,-a+b i,(b+\beta) i$ and $(b-\beta) i$ for $a, b, \beta \in \mathbb{R}$. Then, for every non-negative integers $m_{1}, m_{2}$ we have

$$
\left|f^{\left(m_{1}+m_{2}\right)}(a+b i)\right|^{2} \leq(-1)^{m_{1}+m_{2}} f^{\left(2 m_{1}\right)}(i(b+\beta)) f^{\left(2 m_{2}\right)}(i(b-\beta)) .
$$

Proof. See [10].

Remark 6.3. Observe that the case $m_{1}=m_{2}=0$ in (6.2) is just inequality (5.4) in Proposition 5.6, which as noted at the time requires no regularity on $f$. So in fact inequalities (6.2) may be thought of as the generalization of the basic property of positive definite functions stated in (5.4) to the holomorphic case.

These results may now be used to prove the following complex variable version of Theorem 6.1.

Theorem 6.4. Suppose $S$ is an open codifference set and let $f: S \rightarrow \mathbb{C}$ be a positive definite holomorphic function. If $f^{(2 m)}(i b)=0$ for some nonnegative integer $m$ and some $b \in \mathbb{R}$ with $z=i b \in S$, then $f$ is constant on the open connected component of $S$ containing $i b$.

Proof. See [10].

Remark 6.5. As with previous results in this section, this result may be viewed as an extension to the holomorphic setting of a basic property of positive definite functions. In fact, the special case $m=0$ of Theorem 6.4 is Theorem 5.9 which, as stated at the time, is valid without imposing any regularity on $f$.

The next results illustrate the effect of positivity on the existence and location of the poles of a meromorphic function. In what follows we denote the domain of a complex function by $D(f)$.

Theorem 6.6. Let $\Omega \subset \mathbb{C}$ be an open set and let $S=\operatorname{codiff}(\Omega)$. Suppose $f$ is meromorphic in $S$ and positive definite in $S \cap D(f)$. Then $f$ is holomorphic in $S$.

Proof. See [10]. 
Remark 6.7. Suppose that $f$ is meromorphic in a horizontal strip $S\left(r, \alpha_{1}, \alpha_{2}\right)=\left\{a+b i \in \mathbb{C}:|a|<r, \alpha_{1}<b<\alpha_{2}\right\}$, with $r, \alpha_{1}, \alpha_{2}$ positive real or infinite. If $f$ is positive definite in $S \cap D(f)$, it follows from the fact that $S$ is a codifference set and Theorem 6.6 that $f$ is holomorphic in $S$.

Corollary 6.8. Suppose $f$ is meromorphic in $\mathbb{C}$ and positive definite in its domain. Then $f$ is entire.

Proof. Immediate from Remark 6.7 when $r, \alpha_{1}$ and $\alpha_{2}$ are infinite.

If $f$ is meromorphic in $\mathbb{C}$ and analytic at $z \in D(f)$, we shall henceforth denote the radius of convergence of the Taylor series of $f$ about $z$ by $r(z)$. The well-known dependence of $r(z)$ on the growth rate of the derivatives of $f$ at $z$ allows us to derive the following result from the differential inequalities (6.2).

Lemma 6.9. Let $f$ be a meromorphic function in $\mathbb{C}$. Suppose $f$ is positive definite in $S \cap D(f)$ for some open set $S \subset \mathbb{C}$ and that $\pm a+b i, b \pm \beta i \in$ $S \cap D(f)$ for some $a, b, \beta \in \mathbb{R}$. Then

$$
r^{2}(a+b i) \geq r((b+\beta) i) r((b-\beta) i) .
$$

Two distinct consequences of this lemma may now be stated.

Theorem 6.10. Let $S \subset \mathbb{C}$ be an open set containing $z=i b, b \in \mathbb{R}$. Suppose $f$ is meromorphic in $\mathbb{C}$ and positive definite on $S \cap D(f)$. If $f$ has no poles on the imaginary axis, then $f$ is entire.

Proof. See [10].

Theorem 6.11. Let $\mathcal{L}\left(b_{0}\right)$ be the horizontal line defined by $\mathcal{L}\left(b_{0}\right)=\{z \in$ $\left.\mathbb{C}: z=a+i b_{0}\right\}$, for $b_{0} \in \mathbb{R}$, and let $f$ be a meromorphic function in $\mathbb{C}$. Suppose $f$ is positive definite in $S \cap D(f)$ and that $\mathcal{L}\left(b_{0}\right) \subset S \cap D(f)$. Then $f$ has no poles on the strip $S=\left\{z=a+b i \in \mathbb{C}: a \in \mathbb{R}\right.$ and $\left.\left|b-b_{0}\right|<r\left(b_{0}\right)\right\}$. If $r\left(b_{0}\right)<+\infty$, then at least one of $i\left(b \pm r\left(b_{0}\right)\right)$ is a pole of $f$.

Proof. See [10].

The work described in this paper suggests several lines of future research. For instance, do the poles of a positive definite function $f$ on the imaginary axis determine the existence, location and order of other poles? A preliminary result suggests that much can be said along those lines [3], possibly beginning with the study of horizontal strips and their boundaries as special cases of codifference sets. 


\section{References}

[1] N. Aronszajn, Theory of reproducing kernels. Trans. Amer. Math. Soc. 68, (1950). 337-404.

[2] C. Berg, J. Christensen, P. Ressel, Harmonic analysis on semigroups. SpringerVerlag, GTM 100, New York, 1984.

[3] T. Bisgaard and Z. Sasvári, Characteristic functions and moment sequences. Nova Science Publishing, New York, 2000.

[4] H. Bremermann, Holomorphic continuation of the kernel function and the Bergman metric on several complex variables. Lectures on functions of a complex variable, 349-383. The University of Michigan Press, Ann Arbor, 1955.

[5] J. Buescu, A. Paixão, Positive definite matrices and differentiable reproducing kernel inequalities. J. Math. Anal. Appl. 320 (2006), 279-292.

[6] J. Buescu, A. Paixão, Eigenvalue distribution of positive definite kernels on unbounded domains. Integral Equations Operator Theory 57 (2007), 1, 19-41.

[7] J. Buescu, A. Paixão, A linear algebraic approach to holomorphic reproducing kernels in $\mathbb{C}^{n}$. Linear Algebra Appl. 412 (2006), no. 2-3, 270-290.

[8] J. Buescu, A. Paixão, Positive definite matrices and integral equations on unbounded domains. Differential Integral Equations 19 (2006), no. 2, 189-210.

[9] J. Buescu, A. Paixão, On differentiability and analyticity of positive definite functions. J. Math. Anal. Appl. 375 (2011), no. 1, 336-341.

[10] J. Buescu, A. Paixão, Complex variable positive definite functions. Submitted for publication.

[11] S. Y. Chung, Quasianaliticity of positive definite continuous functions. Publ. RIMS, Kyoto Univ., 38 (2002), 725-733.

[12] S. Y. Chung, S. Y. Yim, Global regularity of positive definite continuous functions. Integr. Transf. Spec. F. 11, 4 (2001), 345-352.

[13] A. Devinatz, On infinitely differentiable positive definite functions. Proc. Amer. Math. Soc. 8 (1957), 3-10.

[14] W. F. Donoghue, Distributions and Fourier transforms. Academic Press, New York, 1969.

[15] D. Dugué, Analyticité et convexité des fonctions caractéristiques. Annales de l'I. H. P. 12, 1 (1951), 45-56.

[16] C. H. Fitzgerald, On analytic continuation to Schlicht functions. Proc. Amer. Math. Soc. 18, 5 (1967), 788-792.

[17] H. Kosaki (2011), Positive definiteness of functions with applications to Operator Norm Inequalities. Mem. Amer. Math. Soc., 212

[18] S. Krantz, H. Parks (2002), A Primer of Real Analytic Functions , $2^{\text {nd }}$ ed., Birkhäuser, Basel, 2002.

[19] E. Lukacs and O. Szasz, On analytic characteristic functions. Pacific J. Math. 3, (1953), 615-625.

[20] C. E. Shin, S. Y. Chung, Analiticity and quasianaliticity of positive definite distributions. J. Math. Kyoto Univ., 44, 4 (2002), 811-817.

[21] E. H. Moore, General Analysis. Memoirs of Amer. Philos. Soc. Part I (1935), Part II (1939).

[22] Z. Sasvári, Positive definite and definitizable functions. Mathematical Topics, 2. Akademie Verlag, Berlin, 1994. 